\title{
RECURRENCE RELATIONS FOR CONNECTION COEFFICIENTS BETWEEN Q-ORTHOGONAL POLYNOMIALS OF DISCRETE VARIABLES IN THE NON-UNIFORM LATTICE $x(s)=q^{2 s} \cdot{ }^{1}$
}

\author{
R. Álvarez-Nodarse ${ }^{2}$ \\ Departamento de Matemáticas. Escuela Politécnica Superior. \\ Universidad Carlos III de Madrid. Butarque 15, 28.911, Leganés, Madrid. \\ A. Ronveaux ${ }^{3}$ \\ Mathematical Physics, Facultés Universitaires Notre-Dame de la Paix, \\ B-5000 Namur, Belgium.
}

Key words and phrases: q-Hahn, q-Meixner, q-Charlier and q-Kravchuk polynomials, discrete polynomials, q-polynomials polynomials.

AMS (MOS, 1991) subject classification: 33D45

\begin{abstract}
We obtain the structure relations for q-orthogonal polynomials in the exponential lattice $q^{2 s}$ and from that we construct the recurrence relation for the connection coefficients between two families of polynomials belonging to the classical class of discrete q-orthogonal polynomials. An explicit example is also given.
\end{abstract}

\section{Introduction.}

Given two families of Polynomials, denoted by $P_{n}(x)$ and $Q_{m}(x)$, of degree exactly equal to respectively $n$ and $m$, the Connection Problem asks to compute the so-called Connection Coefficients $C_{m}(n)$ defined by the relation:

$$
P_{n}(x)=\sum_{m=0}^{n} C_{m}(n) Q_{m}(x) .
$$

When both families are orthogonal with respect to two different measures the Connections Coefficients satisfy a relative simple recurrence relation, but mixing in the $(m, n)$ table three adjacent $m$ and three adjacent $n$ crossing at $(m, n)$.

The first survey on this topic was given by Askey 20 years ago [1]-[2], giving in some cases explicit expression for the Coefficients and discussing also the positivity properties of these Coefficients.

\footnotetext{
${ }^{1}$ October 29, 1996

${ }^{2}$ e-mail renato@dulcinea.uc3m.es Fax:(+431) 6249430

${ }^{3}$ e-mail Andre.Ronveaux@fundp.ac.be Fax $(+3281) 724707$
} 
It was noticed only recently that an additional assumption on the orthogonality measure gives for $C_{m}(n)$ a recurrence only in $m, n$ being fixed. This Orthogonality class is called semi-classical and is very large [11], [7]. The classical (continuous) family: Jacobi, Bessel, Laguerre, Hermite (see for instance [12]) and the classical(discrete) family: Hahn, Kravchuk, Meixner, Charlier (see for instance [13]) are of course included in the semi-classical class. When the orthogonality measure is defined by a weight $\rho(x)$, the semi-classical class covers all weights solution of a linear first order differential (or difference) equation with polynomial coefficients.

The key property inside the semi-classical class, in order to obtain a one index (m) recurrence relation for $C_{m}(n)$, comes from the existence of a so called Structure Relation, linking linearly the derivative (or difference) of $P_{n}(x)$ times a polynomial, to a fixed combination of $P_{k}(x)$.

An algorithm has been given recently building for both discrete and continuous classical families (see [3], [15] and [16] ) the explicit recurrences for $C_{m}(n)$, solving in many cases theses recurrences with the help of Mathematica [20].

Looking for the situation for which a structure relation is known explicitly, we realize that, from the data of Orthogonal Polynomial on the exponential lattice $x(s)=q^{2 s}$ (a small subset of the q-world). Here we need to point out that exits two different point of view in the study of the q-polynomials. The first one, in the framework of the q-basic hypergeometric series [6], [8], [9] and the second, in the framework of the theory of difference equations developed by Nikiforov et al. [12], [13], [14]. In this work we will use the second one because it gives us the possibility to provide an uniform treatment of several classes of orthogonal polynomials and, probably, it is the best way to find further applications.

This paper shows how to apply the technique to a particular (simple) case: the exponential lattice, building first the corresponding Structure Relations.

\section{Structure relations for q-orthogonal polynomials on the exponential lattice $x(s)=q^{2 s}$.}

Let us to start with the study of some general properties of orthogonal polynomials of a discrete variable in non-uniform lattices. Let be

$$
\begin{gathered}
\tilde{\sigma}(x(s)) \frac{\triangle}{\triangle x\left(s-\frac{1}{2}\right)} \frac{\nabla Y(s)}{\nabla x(s)}+\frac{\tilde{\tau}(x(s))}{2}\left[\frac{\triangle Y(s)}{\triangle x(s)}+\frac{\nabla Y(s)}{\nabla x(s)}\right]+\lambda Y(s)=0, \\
\nabla f(s)=f(s)-f(s-1), \triangle f(s)=f(s+1)-f(s),
\end{gathered}
$$

the second order difference equation of hypergeometric type for some lattice function $x(s)$, where $\nabla f(s)=f(s)-f(s-1)$ and $\triangle f(s)=f(s+1)-f(s)$ denote the backward and forward finite difference quotients, respectively. Here $\tilde{\sigma}(x)$ and $\tilde{\tau}(x)$ are polynomials in $x(s)$ of degree at most 2 and 1 , respectively, and $\lambda$ is a constant. 
The previous equation (1) can be obtained from the classical hypergeometric equation

$$
\tilde{\sigma}(x) y^{\prime \prime}(x)+\tilde{\tau}(x) y^{\prime}(x)+\lambda y(s)=0,
$$

via the discretization of the first and second derivatives $y^{\prime}$ and $y^{\prime \prime}$ in an apropiate lattice [12], [13]. It is better to rewrite (1) in the equivalent form (see [13] and [14])

$$
\begin{gathered}
\sigma(s) \frac{\triangle}{\triangle x\left(s-\frac{1}{2}\right)} \frac{\nabla Y(s)}{\nabla x(s)}+\tau(s) \frac{\triangle Y(s)}{\triangle x(s)}+\lambda Y(s)=0, \\
\sigma(s)=\tilde{\sigma}(x(s))-\frac{1}{2} \tilde{\tau}(x(s)) \triangle x\left(s-\frac{1}{2}\right), \quad \tau(s)=\tilde{\tau}(x(s)) .
\end{gathered}
$$

The q-orthogonal polynomials $P_{n}(x(s))_{q} \equiv P_{n}(s)_{q}$ on the exponential lattice $x(s)=$ $q^{2 s}$ are, for given functions $\sigma(s)$ and $\tau(s)$, the polynomial (in powers of $x(s)=q^{2 s}$ ) solution of the second order difference equation (2).

The k-order difference derivative of the polynomials $P_{n}(x(s))_{q}$, defined by

$$
v_{k n}(s)=\frac{\triangle}{\triangle x_{k-1}(s)} \frac{\triangle}{\triangle x_{k-2}(s)} \ldots \frac{\triangle}{\triangle x(s)}\left[P_{n}(x(s))_{q}\right] \equiv \triangle^{(k)}\left[P_{n}(x(s))_{q}\right],
$$

and

$$
x_{m}(s)=x\left(s+\frac{m}{2}\right),
$$

also satisfy the difference equation of hypergeometric type of the form

$$
\sigma(s) \frac{\triangle}{\triangle x_{k}\left(s-\frac{1}{2}\right)}\left[\frac{\nabla v_{k n}(s)}{\nabla x_{k}(s)}\right]+\tau_{k}(s) \frac{\triangle v_{k n}(s)}{\triangle x_{k}(s)}+\mu_{k} v_{k n}(s)=0,
$$

where (see [13], page 62, Equation (3.1.29))

$$
\tau_{k}(s)=\frac{\sigma(s+k)-\sigma(s)+\tau(s+k) \triangle x\left(s+k-\frac{1}{2}\right)}{\triangle x_{k-1}(s)},
$$

and

$$
\mu_{k}=\lambda_{n}+\sum_{m=0}^{k-1} \frac{\triangle \tau_{m}(s)}{\triangle x_{m}(s)} .
$$

These polynomial solutions denoted by $P_{n}(x(s))_{q} \equiv P_{n}(s)_{q}$ satisfy the orthogonality property

$$
\sum_{s_{i}=a}^{b-1} P_{n}\left(x\left(s_{i}\right)\right)_{q} P_{m}\left(x\left(s_{i}\right)\right)_{q} \rho\left(s_{i}\right) \triangle x\left(s_{i}-\frac{1}{2}\right)=\delta_{n m} d_{n}^{2},
$$

where $\rho(x)$ is some non-negative function (weight-function), i.e.,

$$
\rho\left(s_{i}\right) \triangle x\left(s_{i}-\frac{1}{2}\right)>0 \quad\left(a \leq s_{i} \leq b-1\right),
$$

supported in a countable subset of the real line $[a, b](a, b$ can be $\pm \infty)$. The functions $\rho(s)$ and $\rho_{k}(s)$ are the solutions of the Pearson-type difference equations ([13], Eq.(3.2.9) and (3.2.10) page 64$)$

$$
\frac{\triangle}{\triangle x\left(s-\frac{1}{2}\right)}[\sigma(s) \rho(s)]=\tau(s) \rho(s),
$$


and

$$
\frac{\triangle}{\triangle x_{k}\left(s-\frac{1}{2}\right)}\left[\sigma(s) \rho_{k}(s)\right]=\tau_{k}(s) \rho_{k}(s)
$$

and $\rho(s)$ satisfy the condition [14]:

$$
\left.\sigma(s) \rho(s) x^{k}\left(s-\frac{1}{2}\right)\right|_{s=a, b}=0, \quad \forall k, l \in \mathbb{N} \quad(\mathbb{N}=\{0,1,2, \ldots\}) .
$$

In (4) $d_{n}^{2}$ denotes the square of the norm of the corresponding orthogonal polynomials.

The q-orthogonal polynomials satisfy a three term recurrence relations (TTRR) of the form

$$
x(s) P_{n}(s)_{q}=\alpha_{n} P_{n+1}(s)_{q}+\beta_{n} P_{n}(s)_{q}+\gamma_{n} P_{n-1}(s)_{q},
$$

with the initial conditions

$$
P_{-1}(s)_{q}=0, \quad P_{0}(s)_{q}=1 \text {. }
$$

It is well known [13]-[14], that the polynomial solutions of equation (2), denoted by $P_{n}(x(s))_{q}$, are uniquely determined, up to a normalizing factor $B_{n}$, by the difference analog of the Rodrigues formula (see [13] page $66 \mathrm{Eq} .(3.2 .19)$ ):

$$
P_{n}(s)_{q}=\frac{B_{n}}{\rho(s)} \nabla_{n}^{(n)}\left[\rho_{n}(s)\right] \quad \nabla_{n}^{(n)}=\frac{\nabla}{\nabla x_{1}(s)} \frac{\nabla}{\nabla x_{2}(s)} \cdots \frac{\nabla}{\nabla x_{n}(s)}\left[\rho_{n}(s)\right],
$$

where $\rho_{n}(s)=\rho(n+s) \prod_{k=1}^{n} \sigma(s+k)$. These solutions correspond to some values of $\lambda_{n}$ - the eigenvalues of equation (2), which is computed from ( see [13], page 104 and [14])

$$
\lambda_{n}=-\frac{1}{2}[n]_{q}\left\{\left(q^{n-1}+q^{-n+1}\right) \tilde{\tau}^{\prime}+[n-1]_{q} \tilde{\sigma}^{\prime \prime}\right\}
$$

where $\tilde{\sigma}(s)=\sigma(s)+\frac{1}{2} \tilde{\tau}(s) \triangle x\left(s-\frac{1}{2}\right)$ and $\tilde{\tau}(s)=\tau(s)($ see Eq. (2)).

Here $[n]_{q}$ denotes the so called $q$-numbers

$$
[n]_{q}=\frac{q^{n}-q^{-n}}{q-q^{-1}}=\frac{\sinh (h n)}{\sinh (h)}, \quad q=e^{h}
$$

\subsection{The first structure relation for the q-polynomials in the lattice $x(s)=q^{2 s}$.}

Let us now try to obtain a structure relation for the q-polynomials in the exponential lattice $x(s)=q^{2 s}$. (For the linear lattice see [13] Eq.(2.2.10) page 24.)

First of all, we rewrite the Rodrigues equation (8) in another form. We will use the linearity of the operator $\nabla_{n}^{(n)}$, as well as the identity

$$
\nabla x_{k}(s)=q^{k} \nabla x(s)
$$

Then, a straightforward calculation gives us 


$$
P_{n}(s)_{q}=\frac{q^{-\frac{n(n+1)}{2}} B_{n}}{\rho(s)}\left[\frac{\nabla}{\nabla x(s)}\right]^{n}\left[\rho_{n}(s)\right], \quad\left[\frac{\nabla}{\nabla x(s)}\right]^{n}=\overbrace{\frac{\nabla}{\nabla x(s)} \cdots \frac{\nabla}{\nabla x(s)}}^{\text {n-times }} .
$$

Now, from formulas (5) and (10) we find

$$
\frac{\nabla \rho_{n+1}(s)}{\nabla x_{n+1}(s)}=\frac{\nabla\left[\rho_{n}(s+1) \sigma(s+1)\right]}{\nabla x_{n}\left(s+\frac{1}{2}\right)}=\frac{\triangle\left[\sigma(s) \rho_{n}(s)\right]}{\triangle x_{n}\left(s-\frac{1}{2}\right)}=\tau_{n}(s) \rho_{n}(s) .
$$

Then by using the Rodrigues formula (8) we obtain

$$
\begin{gathered}
P_{n+1}(s)_{q}=\frac{B_{n+1}}{\rho(s)} \nabla_{n+1}^{(n+1)}\left[\rho_{n}(s)\right]=\frac{B_{n+1}}{\rho(s)} \nabla_{n}^{(n)} \frac{\nabla \rho_{n+1}(s)}{\nabla x_{n+1}(s)}= \\
=\frac{B_{n+1}}{\rho(s)} \nabla_{n}^{(n)}\left[\tau_{n}(s) \rho_{n}(s)\right]=q^{-\frac{n(n+1)}{2}} \frac{B_{n+1}}{\rho(s)}\left[\frac{\nabla}{\nabla x(s)}\right]^{n}\left[\tau_{n}(s) \rho_{n}(s)\right] .
\end{gathered}
$$

In order to obtain an expression for $\left[\frac{\nabla}{\nabla x(s)}\right]^{n}\left[\tau_{n}(s) \rho_{n}(s)\right]$ we successively apply the formula $\nabla f(s) g(s)=f(s) \nabla g(s)+g(s-1) \nabla f(s)$, as well as formulas

$$
\frac{\triangle \tau_{n}(s)}{\triangle x(s)}=q^{n} \tau_{n}^{\prime}, \quad\left[\frac{\nabla}{\nabla x(s-1)}\right]^{n}=q^{2 n}\left[\frac{\nabla}{\nabla x(s)}\right]^{n} .
$$

Then, Eq. (11) gives us the following

$$
\begin{aligned}
P_{n+1}(s)_{q}= & \frac{q^{-\frac{n(n+1)}{2}} B_{n+1}}{\rho(s)} \times \\
& \times\left(\tau_{n}(s)\left[\frac{\nabla}{\nabla x(s)}\right]^{n}\left[\rho_{n}(s)\right]+q^{2 n-1}[n]_{q} \tau_{n}^{\prime}\left[\frac{\nabla}{\nabla x(s)}\right]^{n-1}\left[\rho_{n}(s-1)\right]\right) .
\end{aligned}
$$

Using the Rodrigues formula for the difference derivative of the polynomial ([13], Eq. (3.2.18) page 66) we find (notice that $\triangle x(s-1)=q^{-2} \triangle x(s)$ ):

$$
\begin{aligned}
\frac{\nabla P_{n}(s)_{q}}{\nabla x(s)} & =\frac{\triangle P_{n}(s-1)_{q}}{\triangle x(s-1)}=\frac{-q^{-\frac{(n-1)(n+2)}{2}} \lambda_{n} B_{n}}{\sigma(s) \rho(s)}\left[\frac{\nabla}{\nabla x(s-1)}\right]^{n-1}\left[\rho_{n}(s-1)\right]= \\
& =\frac{-q^{-\frac{(n-1)(n-2)}{2}} \lambda_{n} B_{n}}{\sigma(s) \rho(s)}\left[\frac{\nabla}{\nabla x(s)}\right]^{n-1}\left[\rho_{n}(s-1)\right] .
\end{aligned}
$$

Therefore, equation (12) can be rewritten in the form

$$
P_{n+1}(s)_{q}=\frac{B_{n+1} \tau_{n}(s)}{B_{n}} P_{n}(s)_{q}-\frac{[n]_{q} B_{n+1} \tau_{n}^{\prime} \sigma(s)}{\lambda_{n} B_{n}} \frac{\nabla P_{n}(s)_{q}}{\nabla x(s)}
$$

and then, the following differentiation formula holds

$$
\sigma(s) \frac{\nabla P_{n}(s)_{q}}{\nabla x(s)}=\frac{\lambda_{n}}{[n]_{q} \tau_{n}^{\prime}}\left[\tau_{n}(s) P_{n}(s)_{q}-\frac{B_{n}}{B_{n+1}} P_{n+1}(s)_{q}\right] .
$$


If we now use the power expansion of $\tau_{n}(s)$, i.e., $\tau_{n}(s)=\tau_{n}^{\prime} x_{n}(s)+\tau_{n}(0)=\tau_{n}^{\prime} q^{n} x(s)+$ $\tau_{n}(0)$ and the TTRR $(7)$ we obtain the first structure relation

$$
\sigma(s) \frac{\nabla P_{n}(s)_{q}}{\nabla x(s)}=\tilde{S}_{n} P_{n+1}(s)_{q}+\tilde{T}_{n} P_{n}(s)_{q}+\tilde{R}_{n} P_{n-1}(s)_{q},
$$

where

$$
\begin{aligned}
& \tilde{S}_{n}=\frac{\lambda_{n}}{[n]_{q}}\left[q^{n} \alpha_{n}-\frac{B_{n}}{\tau_{n}^{\prime} B_{n+1}}\right], \\
& \tilde{T}_{n}=\frac{\lambda_{n}}{[n]_{q}}\left[q^{n} \beta_{n}-\frac{\tau_{n}(0)}{\tau_{n}^{\prime}}\right], \\
& \tilde{R}_{n}=\frac{\lambda_{n} q^{n} \gamma_{n}}{[n]_{q}} .
\end{aligned}
$$

\subsection{The second structure relation for the q-polynomials in the lat-} tice $x(s)=q^{2 s}$.

Let us try to obtain now the second structure relation. Firstly, we notice that

$$
\triangle \frac{\nabla P_{n}(s)_{q}}{\nabla x(s)}=\frac{\triangle P_{n}(s)_{q}}{\triangle x(s)}-\frac{\nabla P_{n}(s)_{q}}{\nabla x(s)} .
$$

Then, by using the difference equation (2)

$$
\begin{aligned}
\sigma(s) \frac{\nabla P_{n}(s)_{q}}{\nabla x(s)} & =\sigma(s) \frac{\triangle P_{n}(s)_{q}}{\triangle x(s)}-\sigma(s) \triangle \frac{\nabla P_{n}(s)_{q}}{\nabla x(s)}= \\
& =\left[\sigma(s)+\tau(s) \triangle x\left(s-\frac{1}{2}\right)\right] \frac{\triangle P_{n}(s)_{q}}{\triangle x(s)}+\lambda_{n} \triangle x\left(s-\frac{1}{2}\right) P_{n}(s)_{q} .
\end{aligned}
$$

and (14) we find

$$
\begin{aligned}
{[\sigma(s)+} & \left.\tau(s) \triangle x\left(s-\frac{1}{2}\right)\right] \frac{\triangle P_{n}(s)_{q}}{\triangle x(s)}=\tilde{S}_{n} P_{n+1}(s)_{q}+ \\
& +\left(\tilde{T}_{n}-\lambda_{n} \triangle x\left(s-\frac{1}{2}\right)\right) P_{n}(s)_{q}+\tilde{R}_{n} P_{n-1}(s)_{q}
\end{aligned}
$$

Now, taking into account that $\triangle x\left(s-\frac{1}{2}\right)=\left(q-q^{-1}\right) x(s)$, and using the TTRR (7) we finally obtain the second structure relation

$$
\left[\sigma(s)+\tau(s) \triangle x\left(s-\frac{1}{2}\right)\right] \frac{\triangle P_{n}(s)_{q}}{\triangle x(s)}=S_{n} P_{n+1}(s)_{q}+T_{n} P_{n}(s)_{q}+R_{n} P_{n-1}(s)_{q},
$$

where

$$
\begin{aligned}
& S_{n}=\tilde{S}_{n}-\left(q-q^{-1}\right) \lambda_{n} \alpha_{n}, \\
& T_{n}=\tilde{T}_{n}-\left(q-q^{-1}\right) \lambda_{n} \beta_{n}, \\
& R_{n}=\tilde{R}_{n}-\left(q-q^{-1}\right) \lambda_{n} \gamma_{n} .
\end{aligned}
$$




\section{$3 \quad$ Recurrence relations for connection coefficients.}

Let us consider two families of q-polynomials $P_{n}(x)$ and $Q_{n}(x)$ belonging to the class of discrete orthogonal polynomials in the exponential lattice $x(s)=q^{2 s}$. Each polynomial $P_{n}(x)$ can be represented as a linear combination of the polynomials $Q_{n}(x)$. In particular

$$
P_{n}(x)=\sum_{m=0}^{n} C_{m}(n) Q_{m}(x) .
$$

For the family $P_{n}(x)$ we will use the notation

1. $\sigma(s), \tau(s)$ and $\lambda_{n}$ for the difference equation (2)

2. $\alpha_{n}, \beta_{n}$ and $\gamma_{n}$ for the TTRR (7) coefficients

3. $S_{n}, R_{n}$ and $T_{n}$ for the second structure relation (17)

and for the $Q_{n}(x)$

1. $\bar{\sigma}(s), \bar{\tau}(s)$ and $\bar{\lambda}_{n}$ for the difference equation (2)

2. $\bar{\alpha}_{n}, \bar{\beta}_{n}$ and $\bar{\gamma}_{n}$ for the TTRR (7) coefficients

3. $\bar{S}_{n}, \bar{R}_{n}$ and $\bar{T}_{n}$ for the second structure relation (17)

Since the polynomials of the family $P_{n}(x)$ are solutions of the second order difference equation (2) the action of the difference operator of second order $\hat{L}$, defined by

$$
\hat{L}=\sigma(s) \frac{\triangle}{\triangle x\left(s-\frac{1}{2}\right)}\left[\frac{\nabla}{\nabla x(s)}\right]+\tau(s) \frac{\triangle}{\triangle x(s)}+\lambda_{n},
$$

on Eq. (19) gives us

$$
\sum_{m=0}^{n} C_{m}(n)\left[\sigma(s) \frac{\triangle}{\triangle x\left(s-\frac{1}{2}\right)}\left[\frac{\nabla Q_{m}(x)}{\nabla x(s)}\right]+\tau(s) \frac{\triangle Q_{m}(x)}{\triangle x(s)}+\lambda_{n} Q_{m}(x)\right]=0 .
$$

Multiplying by $\bar{\sigma}(s)$ and using

$$
\bar{\sigma}(s) \frac{\triangle}{\triangle x\left(s-\frac{1}{2}\right)}\left[\frac{\nabla Q_{m}(x)}{\nabla x(s)}\right]=-\bar{\tau}(s) \frac{\triangle Q_{m}(x)}{\triangle x(s)}-\bar{\lambda}_{n} Q_{m}(x),
$$

we obtain the relation

$$
\sum_{m=0}^{n} C_{m}(n)\left[(\tau(s) \bar{\sigma}(s)-\bar{\tau}(s) \sigma(s)) \frac{\triangle Q_{m}(x)}{\triangle x(s)}+\left(\lambda_{n} \bar{\sigma}(s)-\sigma(s) \bar{\lambda}_{m}\right) Q_{m}(x)\right]=0 .
$$

In order to eliminate $\frac{\triangle Q_{m}(x)}{\triangle x(s)}$, we multiply (21) by $\bar{\sigma}(s)+\bar{\tau}(s) \triangle x\left(s-\frac{1}{2}\right)$ and use the second structure relation (17) for the $Q_{m}(x)$ family, obtaining

$$
\begin{gathered}
\sum_{m=0}^{n} C_{m}(n)\left[(\tau(s) \bar{\sigma}(s)-\bar{\tau}(s) \sigma(s))\left(\bar{S}_{m} Q_{m+1}(x)+\bar{R}_{m} Q_{m-1}(x)+\bar{T}_{m} Q_{m}(x)\right)+\right. \\
\left.+\left(\bar{\sigma}(s)+\bar{\tau}(s) \triangle x\left(s-\frac{1}{2}\right)\right)\left(\lambda_{n} \bar{\sigma}(s)-\sigma(s) \bar{\lambda}_{m}\right) Q_{m}(x)\right]=0 .
\end{gathered}
$$


The last step consists to expand the remaining terms of type $\bar{\sigma}^{2}(s) Q_{m}(x), \bar{\sigma}(s) \sigma(s) Q_{m}(x)$, $\sigma(s) \bar{\tau}(s) Q_{m}(x)$ and $\bar{\sigma}(s) \tau(s) Q_{m}(x)$ in linear combination of $Q_{m}(x)$ by using the TTRR (7) repeatedly for the $Q_{m}(x)$ family.

After this process, (22) reduces to

$$
\sum_{m=0}^{N} M_{m}\left[C_{0}(n), C_{1}(n), \ldots, C_{n}(n)\right] Q_{m}(x)
$$

where $N=\max \{n+\operatorname{deg} \sigma+\operatorname{deg}(\bar{\sigma}), n+2 \operatorname{deg}(\bar{\sigma}), n+1+\operatorname{deg}(\bar{\sigma})+\operatorname{deg}(\tau), n+1+$ $\operatorname{deg}(\bar{\tau})+\operatorname{deg}(\sigma), 1+\operatorname{deg}(\bar{\tau})+\operatorname{deg}(\bar{\sigma})\}$.

Taking into account the linear independence of the family $Q_{m}(x)$ we obtain the linear system

$$
M_{m}\left[C_{0}(n), C_{1}(n), \ldots, C_{n}(n)\right]=0 .
$$

These relations contain (linearly) several connection coefficients $C_{i}(n)$ depending essentially on the degrees of $\sigma(s)$ and $\bar{\sigma}(s)$. In the most general situation they are polynomials of second degree in $x(s)=q^{2 s}$. In this case we obtain a relation of the following type the linear system we are looking for

$$
M_{m}\left[C_{m+4}(n), \ldots, C_{m-4}(n)\right]=0,
$$

which is valid for $n$ greater or equal than the number of initial conditions needed to start the recursion $(n \geq 8)$. Notice that for $(n<8)$ the system also gives the solution, but not in a recurrent way.

Notice that for the q-Hahn, q-Meixner, q-Charlier and q-Kravchuk polynomials, as it is show in [13], table $\mathbf{3 . 3}$, page 95 , the $\sigma(s)$ is a polynomial of second degree in $x(s)=q^{2 s}$. This implies that for such polynomials the recurrence relations for the connection coefficient all are of the form (25). Again we want to remark that we are follow the notation introduced by Nikiforov et al. [13].

\section{Recurrence relations for connection coefficients: A simple example.}

As we have noticed in the previous section the recurrence relation for connection coefficients for different classes of q-polynomials are too large (8-terms). Here we will analyze a more simple case. Firstly, notice that in the previous algorithm we have not used the orthogonality property of the polynomials $P_{n}$, and only that they satisfy a difference equation. On the other hand, for the polynomials $Q_{m}$ we need to have structure relations as well as three term recurrence relations. Let us to show and example in which we will decompose a set of polynomials $P_{n}(s)$, satisfying a certain difference equation of first order in the lattice $x(s)=q^{2 s}$, as a linear combination of the orthogonal q-polynomials defined in the same lattice, i.e., the q-Hahn, q-Meixner, q-Kravchuk and q-Charlier orthogonal polynomials (see [13], [4] and [17]) 
Let us define the quantities $(s)_{q}$ and $\left(s_{n}\right)_{q}$, defined by

$$
(s)_{q}=\frac{q^{2 s}-1}{q^{2}-1}=q^{s-1}[s]_{q}
$$

and

$$
\left(s_{n}\right)_{q}=(s)_{q}(s-1)_{q} \cdots(s-n+1)_{q}=\prod_{k=0}^{n-1} \frac{q^{2 s+2 k}-1}{q^{2}-1}
$$

The quantities $\left(s_{n}\right)_{q}$ are closely related to the $q$-Stirling numbers $\tilde{S}_{q^{2}}(n, k), s_{q^{2}}^{*}(n, k)$ [21] by formulas

$$
(s)_{q}^{n}=\sum_{k=0}^{n} \tilde{S}_{q^{2}}(n, k)\left(s_{k}\right)_{q}, \quad\left(s_{n}\right)_{q}=\sum_{k=0}^{n} s_{q^{2}}^{*}(n, k)(s)_{q}^{k}
$$

and satisfy the following two difference equations (here, as before, $x(s)=q^{2 s}$ )

$$
\left(q^{2 s}-1\right) \frac{\nabla\left(s_{n}\right)_{q}}{\nabla x(s)}-q^{-n+1}[n]_{q}\left(s_{n}\right)_{q}=0
$$

and

$$
\left(q^{2 s-2 n+2}-1\right) \frac{\triangle\left(s_{n}\right)_{q}}{\triangle x(s)}-q^{-n+1}[n]_{q}\left(s_{n}\right)_{q}=0 .
$$

Since $\left(s_{n}\right)_{q}$ is a polynomial in $x(s)=q^{2 s}$, it can be represented as a linear combination of the polynomials $Q_{m}(x)$, the q-polynomials in the exponential lattice. In particular

$$
\left(s_{n}\right)_{q}=\sum_{m=0}^{n} C_{m}(n) Q_{m}(x) .
$$

Let us to obtain the recurrence relation for the connection coefficients $C_{m}(n)$ between the $\left(s_{n}\right)_{q}$ and the q-Charlier, q-Meixner or q-Kravchuk. (For q-Hahn polynomials we will consider it separately). In order to do that we apply the operator

$$
\tilde{\mathcal{L}}=\left(q^{2 s}-1\right) \frac{\nabla}{\nabla x(s)}-q^{-n+1}[n]_{q}
$$

to both sides of $(31)$. Using formula $(29)\left(\tilde{\mathcal{L}}\left(s_{n}\right)_{q}=0\right)$ and multiplying by $q^{2 s}$ we obtain the following expression

$$
0=\sum_{m=0}^{n} C_{m}(n)\left\{q^{2 s}\left(q^{2 s}-1\right) \frac{\nabla Q_{m}(x)}{\nabla x(s)}-q^{-m+1}[m]_{q} q^{2 s} Q_{m}(x)\right\} .
$$

Taking into account that for q-Charlier, q-Meixner and q-Kravchuk the $\sigma(s)$ function in (2) coincide with $q^{2 s}\left(q^{2 s}-1\right)$ and applying the structure relation (14) and the TTRR ( 7 ) to the previous expression we find

$$
0=\sum_{m=0}^{n} C_{m}(n)\left\{A_{m} Q_{m+1}(x)+B_{m} Q_{m}(x)+\Gamma_{m} Q_{m-1}(x)\right\},
$$

from where we obtain the following TTRR for the connection coefficients $C_{m}(n)$

$$
A_{m-1} C_{m-1}(n)+B_{m} C_{m}(n)+\Gamma_{m+1} C_{m+1}(n)=0,
$$


where

$$
\begin{aligned}
A_{m-1} & =\tilde{S}_{m-1}-q^{-m+2}[m-1]_{q} \alpha_{m-1}= \\
& =\frac{\lambda_{m-1}}{[m-1]_{q}}\left[q^{m-1} \alpha_{m-1}-\frac{B_{m-1}}{\tau_{m-1}^{\prime} B_{m}}\right]-q^{-m+2}[m-1]_{q} \alpha_{m-1}, \\
B_{m} & =\tilde{T}_{m}-q^{-m+1}[m]_{q} \beta_{m}= \\
& =\frac{\lambda_{m-1}}{[m-1]_{q}}\left[q^{m} \beta_{m}-\frac{\tau_{m}(0)}{\tau_{m}^{\prime}}\right]-q^{-m+1}[m]_{q} \beta_{m}, \\
\Gamma_{m+1} & =\tilde{R}_{m+1}-q^{-m} \gamma_{m+1}=\frac{\lambda_{m+1} q^{m+1} \gamma_{m+1}}{[m+1]_{q}}-q^{-m}[m+1]_{q} \gamma_{m+1} .
\end{aligned}
$$

In order to obtain the recurrence relation for connection coefficients in the q-Hahn case we apply the operator $\tilde{\mathcal{L}}(32)$ to both sides of (31). Taking into account that $\tilde{\mathcal{L}}\left(s_{n}\right)_{q}=0$ and multiplying by $q^{2 \alpha+2 N}-q^{2 s}$ we obtain the following expression

$$
0=\sum_{m=0}^{n} C_{m}(n)\left\{\left(q^{2 \alpha+2 N}-q^{2 s}\right)\left(q^{2 s}-1\right) \frac{\nabla Q_{m}(x)}{\nabla x(s)}-q^{-m+1}[m]_{q} q^{2 s} Q_{m}(x)\right\} .
$$

Taking into account that for q-Hahn the $\sigma(s)$ function in (2) coincide with $\left(q^{2 \alpha+2 N}-\right.$ $\left.q^{2 s}\right)\left(q^{2 s}-1\right)$ (see [17]) and using the structure relation (14) and the TTRR (7) we obtain the same expression (34) as before for the TTRR for the connection coefficients $C_{m}(n)$, where now

$$
\begin{aligned}
A_{m-1} & =\tilde{S}_{m-1}+q^{-m+2}[m-1]_{q} \alpha_{m-1}= \\
& =\frac{\lambda_{m-1}}{[m-1]_{q}}\left[q^{m-1} \alpha_{m-1}-\frac{B_{m-1}}{\tau_{m-1}^{\prime} B_{m}}\right]+q^{-m+2}[m-1]_{q} \alpha_{m-1}, \\
B_{m} & =\tilde{T}_{m}+q^{-m+1}[m]_{q} \beta_{m}-[m]_{q} q^{2 N+2 \alpha-m+1}= \\
& =\frac{\lambda_{m-1}}{[m-1]_{q}}\left[q^{m} \beta_{m}-\frac{\tau_{m}(0)}{\tau_{m}^{\prime}}\right]+q^{-m+1}[m]_{q} \beta_{m}-[m]_{q} q^{2 N+2 \alpha-m+1}, \\
\Gamma_{m+1} & =\tilde{R}_{m+1}+q^{-m} \gamma_{m+1}=\frac{\lambda_{m+1} q^{m+1} \gamma_{m+1}}{[m+1]_{q}}+q^{-m}[m+1]_{q} \gamma_{m+1} .
\end{aligned}
$$

\subsection{The three term recurrence relation for connection coefficients of the q-powers $\left(s_{n}\right)_{q}$ and the q-Meixner polynomials $m_{n}^{\gamma, \mu}(s, q)$.}

Here we will calculate the coefficients $A_{m-1}, B_{m}$ and $\Gamma_{m+1}$ of the three term recurrence relation for connection coefficients $C_{m}(n)(34)$ of the q-powers $\left(s_{n}\right)_{q}$ and the q-Meixner polynomials $m_{n}^{\gamma, \mu}(s, q)$, i.e.,

$$
\left(s_{n}\right)_{q}=\sum_{k=0}^{n} C_{m}(n) m_{k}^{\gamma, \mu}(s, q)
$$


The main data for the q-Meixner polynomials are provided in [4]. In our work we will use monic polynomials, i.e., the leading coefficient $a_{n}=1$. In the Table I, at the end of this section, we provide the quantities needed for our calculations. (For more details see [4] and [13]). We want to point out that these monic q-Meixner polynomials $m_{n}^{\gamma, \mu}(s, q)$ [4] are connected with the monic little q-Jacobi polynomials $p_{n}(x ; a, b \mid q)[6],[8]$ by the relation

$$
m_{n}^{\gamma, \mu}(s, q)=p_{n}\left(q^{2 s} ; \mu, q^{2 \gamma-2} \mid q^{2}\right) .
$$

If we now apply formulas (15) and (14) we obtain for q-Meixner polynomials the structure relation

$$
\sigma(s) \frac{\nabla m_{n}^{\gamma, \mu}(s, q)}{\nabla x(s)}=\tilde{S}_{n} m_{n+1}^{\gamma, \mu}(s, q)+\tilde{T}_{n} m_{n}^{\gamma, \mu}(s, q)+\tilde{R}_{n} m_{n-1}^{\gamma, \mu}(s, q),
$$

where

$$
\begin{aligned}
\tilde{S}_{n}= & -q^{\gamma+\theta+1}\left(q^{n}[n+\gamma+\theta]_{q}+[2 n+\gamma+\theta]_{q}\right), \\
\tilde{T}_{n}= & -q^{2 n+\theta+2}[n+\gamma+\theta]_{q}\left(\frac{[n+1]_{q}[n+\theta+1]_{q}}{[2 n+\gamma+\theta+1]_{q}}-\frac{[n]_{q}[n+\theta]_{q}}{[2 n+\gamma+\theta-1]_{q}}\right)- \\
& -\frac{q^{\theta+1}[n+\theta+1]_{q}[n+\gamma+\theta]_{q}}{[2 n+\gamma+\theta+1]_{q}} \\
\tilde{R}_{n}= & -\frac{q^{-2 \gamma+\theta-1}[n]_{q}[\gamma+n-1]_{q}[n+\gamma+\theta]_{q}[n+\gamma+\theta-1]_{q}[n+\theta]_{q}}{[2 n+\gamma+\theta-2]_{q}[2 n+\gamma+\theta-1]_{q}^{2}[2 n+\gamma+\theta]_{q}} .
\end{aligned}
$$

Then, by using (35) we finally find the coefficients $A_{m-1}, B_{m}$ and $\Gamma_{m+1}$

$$
\begin{gathered}
A_{m-1}=-q^{\gamma+\theta+1}\left(q^{m-1}[m+\gamma+\theta-1]_{q}+[2 m+\gamma+\theta-2]_{q}\right)-q^{-m+2}[m-1]_{q}, \\
B_{m}=-\left(\frac{[m+1]_{q}[m+\theta+1]_{q}}{[2 m+\gamma+\theta+1]_{q}}-\frac{[m]_{q}[m+\theta]_{q}}{[2 m+\gamma+\theta-1]_{q}}\right) q^{\theta+1}[2 m+\gamma+\theta]_{q}- \\
-\frac{q^{\theta+1}[m+\gamma+\theta]_{q}[m+\theta+1]_{q}}{[2 m+\gamma+\theta+1]_{q}} \\
\Gamma_{m+1}=-\frac{q^{-m-2 \gamma+\theta+2}[m+1]_{q}[\gamma+m]_{q}[m+\gamma+\theta]_{q}[m+\theta+1]_{q}}{[2 m+\gamma+\theta]_{q}[2 m+\gamma+\theta+1]_{q}[2 m+\gamma+\theta+2]_{q}} .
\end{gathered}
$$


Table I: The main data for q-Meixner Polynomials [4].

\begin{tabular}{|c|c|}
\hline & $m_{n}^{\gamma, \mu}(s, q), \mu=q^{2 \theta}$ \\
\hline$\sigma(s)$ & $q^{2 s}\left(q^{2 s}-1\right)$ \\
\hline$\tau(s)$ & $q^{s+2 \theta+\gamma+2}[s+\gamma]_{q}-q^{s}[s]_{q}$ \\
\hline$\lambda_{n}$ & $-[n]_{q} q^{\gamma+\theta+1}[n+\gamma+\theta]_{q}$ \\
\hline$\tau_{n}^{\prime}$ & $q^{\gamma+\theta+1}[2 n+\gamma+\theta+1]_{q}$ \\
\hline$\tau_{n}(0)$ & $-q^{\theta+1}[n+\theta+1]_{q}$ \\
\hline$\frac{B_{n}}{B_{n+1}}$ & $-q^{\gamma+\theta+1} \frac{[2 n+\gamma+\theta+1]_{q}[2 n+\gamma+\theta]_{q}}{[n+\gamma+\theta]_{q}}$ \\
\hline$\alpha_{n}$ & 1 \\
\hline$\beta_{n}$ & $q^{-\gamma} \frac{[n+1]_{q}[n+\theta+1]_{q}}{[2 n+\gamma+\theta+1]_{q}}-q^{-\gamma} \frac{[n]_{q}[n+\theta]_{q}}{[2 n+\gamma+\theta-1]_{q}}$ \\
\hline$\gamma_{n}$ & $\frac{q^{-n-3 \gamma+2}[n]_{q}[\gamma+n-1]_{q}[n+\gamma+\theta-1]_{q}[n+\theta]_{q}}{[2 n+\gamma+\theta-2]_{q}[2 n+\gamma+\theta-1]_{q}^{2}[2 n+\gamma+\theta]_{q}}$ \\
\hline
\end{tabular}

\section{ACKNOWLEDGMENTS}

The research of the first author was partially supported by Comisión Interministerial de Ciencia y Tecnología (CICYT) of Spain under grant PB 93-0228-C02-01.

\section{References}

[1] R. Askey: Orthogonal Polynomials and Special Functions. Regional Conferences in Applied Mathematics. 21, SIAM, Philadelphia, Pennsylvania, 1975. 
[2] R. Askey: Orthogonal expansions with positive coefficients. Proc. Amer. Матн. Soc. 26, (1965), 1191-1194.

[3] N. M. Atakishiyev, A. Ronveaux and K.B. Wolf: Difference Equation for the Associated Polynomials on the linear lattice. Joun NA OF THEORETICAL AND Mathematical Physics 106(1), (1996) 76-83.

[4] C. Campigotto, Yu.F. Smirnov and S.G. Enikeev. q-Analogue of the Kravchuk and Meixner Orthogonal Polynomials in J. of Comput. And Appl. Math. 57, (1995), 87-97.

[5] T. S. Chihara: An Introduction to Orthogonal Polynomials. Gordon and Breach Publ., New York, 1978.

[6] G.Gasper and M. Rahman: Basic Hypergeometric Series. Cambridge University Press, 1990.

[7] E. Hendriksen and H. van Rossum: Semi-classical orthogonal polynomials. In Proceedings of the Laguerre Symposium - Polynômes orthogonaux et leurs applications. Bar-le-Duc, france, Oct. 1984 (C. Brezinski et al. Eds.) Lecture Notes in Mathematics. Springer-Verlag, New York-Berlin, 1985.

[8] R. Koekoek and R. F. Swarttouw The Askey-scheme of hypergeometric orthogonal polynomials and its q-analogue. Reports of the Faculty of Technical Mathematics and Informatics No. 94-05. Delft University of Technology. Delft 1994.

[9] T.H. Koornwinder: Compact quantum groups and q-special functions. In Representations of Lie groups and quantum groups. V. Baldoni \& M.A. Picardello (eds.) Pitman Research Notes in Mathematics series 311, Longman Scientific \& Technical, (1994), 46-128.

[10] S. Lewanowicz: Quick construction of recurrence relations for the Jacobi coefficients. J. of Comput. and Appl. Мath., 43, (1992), 355-372.

[11] P. Maroni. Prolégomènes à l'etude des polynômes orthogonaux semi-classiques. Ann. Math. Pura ed App. bf 149 (1987) (4), 165-184.

[12] A. F. Nikiforov and V. B. Uvarov: Special Functions of Mathematical Pнуsics. Birkhäuser Verlag, Basel, 1988.

[13] A. F. Nikiforov, S. K. Suslov and V. B. Uvarov: Classical Orthogonal Polynomials of a discrete Variable. Springer Series in Computational Physics. Springer-Verlag,Berlin, 1991.

[14] A. F. Nikiforov and V. B. Uvarov: Polynomial Solutions of hypergeometric type difference Equations and their classification. Integral Transform and SpeCIAL FunCIONS. 1, 223-249, (1993).

[15] A. Ronveaux, A. Zarzo and E. Godoy: Recurrence relation for connection coefficients between two families of orthogonal polynomials. J. OF COMPUT. AND Аррг. Матн., 62, (1995), 67-73. 
[16] A. Ronveaux, S. Belmehdi, E. Godoy and A. Zarzo: Recurrence relation for connection coefficients between classical discrete orthogonal polynomials. Proceedings of the Workshop on Symmetries and Integrability of Difference Equations, C.R.M. and A.M.S. Lecture Notes, 1995. (In press).

[17] Yu. F. Smirnov and A. Del Sol Mesa Orthogonal polynomials of the discrete variable associated with $S U q(2)$ and $S U q(1,1)$ quantum algebras. In International Workshop Summetry Methods in Physics in Memory of Professor Ya.A.Smorodinsky. Eds.A.N.Sissakian, G.S.Pogosyan and S.I.Vinitsky, JINR, E2-94-447, Vol.2, (Dubna 1994), 479-486.

[18] R. Szwarc: Linearization and connection coefficients of orthogonal polynomials. Мн. Матн., 113, (1992), 319-329.

[19] R. Szwarc: Connection coefficients of orthogonal polynomials. CAND. MATH. BulL., 35 (4), (1992) 548-556.

[20] S. Wolfram MATHEMATICA. A system for doing Mathematics by Computer. Addison-Wesley Publishing Co., New York, 1991. Wolfram Research, Inc., Mathematica version 2.2, Wolfram Research, Inc., Champaign, Illinois, (1993).

[21] J. Zeng: The q-Stirling numbers, continued fractions and the q-Charlier and q-Laguerre polynomials. J. Comput. And ApPL. Math., 57, (1995), 413-424. 